\title{
COPD-Behandlung zielt stärker auf Tabakverzicht
}

\author{
Tabakentwöhnung und Verhaltensprävention. Der Gemeinsame Bundesausschuss hat dem DMP \\ COPD eine Auffrischung verpasst. Zudem sollen Ärzte künftig ihre Patienten per Bescheinigung \\ zur Lebensstiländerung motivieren.
}

Die strukturierten Behandlungsprogramme (DMP) sollen künftig nicht mehr isoliert nebeneinander stehen. Das geht aus einer aktuellen Beschlussfassung des Gemeinsamen Bundesausschuss (GBA) hervor.

Damit erfährt das strukturierte Behandlungsprogramm für die chronisch obstruktive Lungenkrankheit (COPD) eine Auffrischung. Stärker als bisher betont die Aktualisierung des DMP die wirksame Behandlung von Komorbiditäten. Genannt werden Diabetes mellitus Typ 2 und kardiovaskuläre Erkrankungen, für die ebenfalls DMP angeboten werden. Auch Depressionen, für die es in absehbarer Zeit ein strukturiertes Behandlungsprogramm geben soll, sind aufgeführt.

An anderer Stelle sollen DMP und Regelversorgung sich verzahnen. So soll zum DMP COPD künftig ein strukturiertes Medikamentenmanagement gehören. Mindestens einmal im Jahr sollen Ärzte sämtliche vom Patienten tatsächlich eingenommenen Arzneien erfassen und auf Wechsel- und Nebenwirkungen hin überprüfen. Der Medikationsplan soll Teil der Patientenakte werden und in verständlicher Form auch dem Patienten ausgehändigt werden. Die neuen DMP-Anforderungen sollen frühestens zum Jahreswechsel in Kraft treten.

Ab Oktober haben auch alle Patienten der Regelversorgung Anspruch auf einen Medikationsplan. Allerdings soll der schon ab drei tatsächlich eingenommener Arzneien ausgehändigt werden.

Die neue Version des DMP zielt noch stärker als bisher auf die Tabakentwöhnung ab. Das Behandlungsprogramm enthält laut GBA hohe Qualitätsanforderungen an Tabakentwöhnungsprogramme.

\section{Präventionsempfehlung „auf Rezept“}

Prävention soll es künftig ,auf Rezept“ geben. Um Patienten zum Verzicht auf Alkohol, Zigaretten oder härtere Drogen zu bewegen, ihnen sportliche Betätigung und andere Ernährungsgewohnheiten nahe zu legen sowie beim Stressabbau zu helfen, können Ärzte ab kommendem Jahr Präventionsempfehlungen auf einer ärztlichen Bescheinigung ausgeben. Die Kassen, so GKV-Spitzenverbandssprecher Florian Lanz, werden den Ärzten ein Formular für ihre Empfehlungen zur Verfügung stellen. Listen mit konkreten Präventionsangeboten soll es aber nicht geben.„Ärzte haben mit dem Ausstellen von Präventionsempfeh- lungen zukünftig eine weitere Möglichkeit, Versicherte zu motivieren, an gesundheitsbezogenen Kursen teilzunehmen", sagte Dr. Harald Deisler, unparteiisches Mitglied des GBA.

Ausdrücklich gilt der Beschluss auch für die Früherkennungsuntersuchungen von Kindern und Jugendlichen: „Die Untersuchungen umfassen, falls medizinisch angezeigt, eine Präventionsempfehlung für Leistungen zur verhaltensbezogenen Prävention (...).“

Krankenkassen müssen diese Empfehlungen berücksichtigen, wenn Patienten Leistungen zur verhaltensbezogenen Prävention beantragen. Derzeit überprüft der GKV-Spitzenverband seinen Präventionsleitfaden. Grundsätzlich lägen die Kriterien und Handlungsfelder für die verhaltensbezogene Prävention aber fest, sagte Florian Lanz.

Anno Fricke

\section{Exazerbationen reduzieren Lungenfunktion vor allem im COPD-Frühstadium}

Akute Exazerbationen einer COPD haben einen irreversiblen Verlust der Lungenfunktion zur Folge, der in den frühen Stadien einer leichten COPD am ausgeprägtesten ist. Dies folgt aus der sogenannten COPDGene 5-year follow-up-cohort-Studie mit 2.861 Rauchern.

Wie die Autorengruppe um Ken Kunisaki vom Minneapolis VA Health Care System beim ATS-Meeting 2016 berichtete, konnte bei 1.937 Patien-

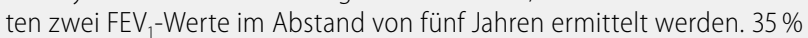
der Patienten hatten mindestens eine Exazerbation bzw. ein respiratorische Komplikation erlebt. Im Falle von COPD-Patienten hatten Exazerbationen einen $\mathrm{FEV}_{1}$-Verlust zur Folge. Am ausgeprägtesten war dieser im Stadium GOLD-1: Leichte Exazerbationen reduzierten die FEV 1 im Schnitt um $21 \mathrm{ml} / J a h r$, schwere Exazerbationen um $75 \mathrm{ml} / \mathrm{Jahr}$.

Im Gegensatz dazu hatten respiratorische Komplikationen bei Patienten ohne Atemwegsobstruktion keinen Abfall der Lungenfunktion zur Folge. Dr. Dirk Einecke

Quelle: ATS-Conference, San Francisco 13-18. Mai 2016 